Tissue-typer instrument. LipostarMSI and in-house ChemomeTricks toolbox for MATLAB software were used for data processing and analysis.

For bottom-up proteomics experiment $\left(n_{a}=10 ; n_{b}=10\right)$, proteins were extracted, separated using SDS-PAGE and digested prior to liquid chromatography separation coupled to an orbitrap MS Q-Exactive HF mass spectrometer. Proteome Discoverer, enrichR and Reactome software were used for data processing and analysis.

Results: MALDI-MSI showed overall differences between OA and OA/TD2 patients based on their specific lipidomic profiles. In particular, sphingomyelin and phosphatidylcholine species were significantly more abundant in OA patients whereas lysolipids such as lysophosphatidylcholine species were mainly present in OA/TD2 patients, providing therefore phenotype-specific OA molecular panels. Additionally, we observed that phosphatidylcholine and sphingomyelin species were more present in the superficial layer of the cartilage whereas lysophosphatidylcholine species were more abundant in the deep layer (Fig. 1A, B). Proteomics experiments applied on cartilage enables the quantification of 114 proteins. Among those, 73 were overexpressed in OA samples whereas 41 were overexpressed in OA/TD2 patients. Among the differentially regulated proteins (Fig. 1C), phospholipase A2 was increased in the diabetic cohort, in line with the elevated level of lysolipids found in the imaging data. Our results also involved the fatty acid omega oxidation and the fatty acid biosynthesis pathways as relevant to explain this deregulation of the lipid metabolism.

Conclusion: MALDI-MSI combined with proteomics experiments showed different profiles between OA and OA/TD2 patients and could be employed for patient classification.

References:

[1] Louati, K., et al., Association between diabetes mellitus and osteoarthritis: systematic literature review and meta-analysis. RMD Open, 2015. 1(1): p. e000077.

[2] Williams, M.F., et al., Type 2 diabetes and osteoarthritis: a systematic review and meta-analysis. J Diabetes Complications, 2016. 30(5): p. 944-50.

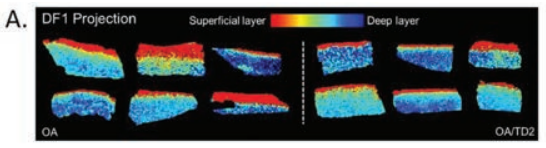

B.

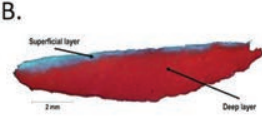

C.

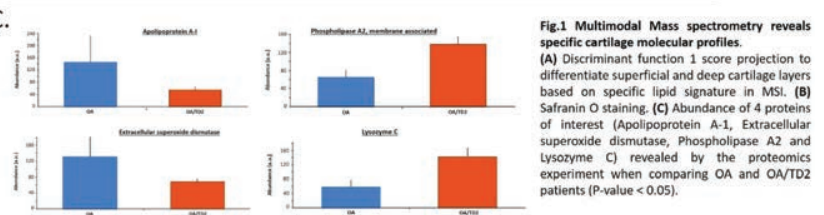

Acknowledgments: The Dutch Province of Limburg and MUMC institutional grant.

Disclosure of Interests: Maxime Eveque: None declared, Pieter Emans Shareholder of: Shareholder and cofounder start-up company Chondropeptix, Grant/ research support from: Institution received grants from STW, ReumaNederland, InSciTE, Consultant of: Consultancy to Kiomed, Speakers bureau: Payment for lectures by Kiomed, Episurf, Britt Claes: None declared, Freek Bouwman: None declared, Ron M A Heeren: None declared, Berta Cillero-Pastor: None declared DOI: 10.1136/annrheumdis-2020-eular.5399

\section{OP0241 IL9 MODULATION OF OSTEOCLASTS DIFFERENTIATION AND IMPACTS ON BONE METABOLISM}

M. Saad ${ }^{1}$, S. Rauber ${ }^{1}$, G. Schett ${ }^{1}$, A. Ramming ${ }^{1} .{ }^{1}$ Department of Internal Medicine 3 - Rheumatology and Immunology, Friedrich-Alexander University (FAU) Erlangen-Nürnberg and Universitätsklinikum Erlangen, Erlangen, Germany

Background: Osteoclasts are multinucleated cells originating from the monocytes/macrophage lineage and require receptor activator of NF-KB ligand (RANK-L) and macrophage-colony-stimulating factor (M-CSF) for their development. They play a major role in bone remodeling by degrading the calcified bone matrix. They are considered as one of the culprits in bone destruction in many inflammatory diseases e.g. rheumatoid arthritis and osteoporosis. In previous work by our group, it was observed that IL- 9 mediated the resolution of inflammation in rheumatoid arthritis and hence protected against bone degradation in animal models. Despite this protection was particularly associated to the resolution of inflammation, our data also supported the hypothesis of a direct signalling of IL-9 to osteoclasts.
Objectives: The aim of this study was to investigate the modulating effect of IL-9 on osteoclasts and on the bone metabolism.

Methods: Osteoclasts differentiation was studied in the mouse models of antigen induced arthritis (AIA) and $\mathrm{KBxN}$ serum induced arthritis (SIA) in the presence and absence of IL- 9 by histomorphometric analysis and microcomputed tomography scans $(\mu \mathrm{CT})$. Osteoclasts were generated from bone marrow derived monocytes of BALB/C mice with M-CSF, RANKL and IL-9, which were added in varying concentrations to induce osteoclast differentiation. Tartrate-resistant acid phosphatase (TRAP) staining was performed to follow the differentiation steps from monocytes into multinucleated osteoclasts and to determine the effects of IL-9 on osteoclastogenesis. Additionally, we performed RNA-seq and seahorse analysis to detect IL-9 dependent, differentially expressed genes and metabolites. Intracellular signaling as induced by IL9R activation was followed by western blot analysis.

Results: IL-9 KO mice showed higher numbers of osteoclasts as compared to wild-type mice in the mouse models of AIA and SIA. Microcomputed tomography showed pronounced loss of the trabecular network and bone volume as signs of inflammation-induced osteopenia in II9-/- mice. We found that osteoclasts express high levels of IL-9R. Next, monocytes were differentiated into osteoclasts in the presence of different concentrations of recombinant IL-9. Cells cultured in the presence of IL-9 showed significantly impaired differentiation into osteoclasts. We observed phosphorylation of STAT3 and STAT5 in cultured osteoclasts in dependency of IL- 9 . Furthermore, the presence of IL- 9 during osteoclast differentiation impacted the gene expression levels of characteristic osteoclast related genes such as NFATc1, Cathepsin K and TRAP. Furthermore, IL-9 showed a major impact on mitochondrial respiration rate and glycolysis as assessed by Seahorse assays.

Conclusion: IL-9 exerted direct effects on osteoclast differentiation and modu lated the expression of several genes that are related to osteoclast differentiation and function.

Disclosure of Interests: mina saad: None declared, Simon Rauber: None declared, Georg Schett Speakers bureau: AbbVie, BMS, Celgene, Janssen, Eli Lilly, Novartis, Roche and UCB, Andreas Ramming Grant/research support from: Pfizer, Novartis, Consultant of: Boehringer Ingelheim, Novartis, Gilead, Pfizer, Speakers bureau: Boehringer Ingelheim, Roche, Janssen DOI: 10.1136/annrheumdis-2020-eular.1676

\section{OP0242 META-ANALYSIS OF SINGLE-CELL RNA SEQUENCING DATA OF THE SYNOVIUM TO DEFINE SYNOVIAL FIBROBLAST PHENOTYPES ACROSS JOINT LOCATION AND DISEASE}

R. Micheroli ${ }^{1}$, M. Frank-Bertoncelj ${ }^{1}$, S. G. Edalat ${ }^{1}$, K. Klein ${ }^{1}$, T. Kuret $^{1,2}$, K. Buerki ${ }^{1}$, A. Ciurea ${ }^{1}$, O. Distler ${ }^{1}$, C. Ospelt ${ }^{1}{ }^{1}$ Center of Exp. Rheumatology, Dep. of Rheumatology, University Hospital Zurich, Zurich, Switzerland; ${ }^{2}$ University Medical Center Ljubljana, Department of Rheumatology, Ljubljana, Slovenia

Background: Up to now, three groups used single cell RNA sequencing (scRNA-seq) to analyse the synovium in arthritis using different methods and material to measure RNA expression on a single cell level: Ref. 1 used unsorted dissociated synovial cells and a droplet based method; Refs 2 and 3 performed scRNA-seq on sorted cell populations.

Objectives: The aim of this study was to perform a meta-analysis of scRNA-seq data of the synovium in arthritis: 1) to define synovial fibroblast (SF) phenotypes, 2 ) to confirm differences across SF clusters between rheumatoid arthritis (RA) and osteoarthritis (OA) and 3 ) to analyse joint specific differences between SF phenotypes.

Methods: In addition to the available count matrices [1-3], we used unsorted dissociated synovial cells from three patients with undifferentiated arthritis (UA) with a droplet-based method (10x Genomics). We followed a standard protocol [4] to integrate the datasets into a shared space, even in the presence of extensive technical and/or biological differences ("batch-corrected"). SF were selected as previously described (PDPN+, ISLR+, COL1A2+, PTPRC-) [1-3]. We used a minimum log2 FC of 0.25 for average expression of genes in a cluster relative to the average expression in all other clusters combined to define marker genes. $\mathrm{R}$ with Seurat, Monocle and clusterProfiler packages were used for scRNA-seq analysis, pseudotime trajectory analysis and pathway enrichement analysis, respectively. Quantitative PCR (qPCR) (n=6-14 per location and disease), immunohistochemistry (IHC) and Krenn synovitis score ( $n=5-15$ per location and disease) were performed according to standard protocols.

Results: Data from 29 RA, 3 UA and 6 OA patients were analysed. From a tota of $29^{\prime} 448$ cells, we identified $14^{\prime} 787$ (50\%) with a fibroblast phenotype. Of those we determined 5 subpopulations (Fig. 1): 1) THY1 ${ }^{-} C D 55^{\text {hi }}$ fibroblasts with high 